\title{
Aprendizaje-servicio y tecnologías digitales: un desafío para los espacios virtuales de aprendizaje
}

\section{(Service-Learning and digital environment of learning: innovative challenges for higher education)}

\author{
Juan García-Gutiérrez \\ Marta Ruiz-Corbella \\ Universidad Nacional de Educación a Distancia, UNED (España) \\ Coordinadores del Monográfico
}

DOI: http://dx.doi.org/10.5944/ried.23.1.25390

\section{Cómo referenciar este artículo:}

García-Gutiérrez, J., y Ruiz-Corbella, M. (2020). Aprendizaje-servicio y tecnologías digitales: un desafío para los espacios virtuales de aprendizaje. RIED. Revista Iberoamericana de Educación a Distancia, 23(1), pp. 31-42. doi: http:// dx.doi.org/10.5944/ried.23.1.25390

\section{Resumen}

Las tecnologías de la información y la comunicación han transformado nuestra forma de ver y actuar en el mundo, lo que ha derivado a que también en el sector educativo se haya experimentado una sacudida sin precedentes. Nadie cuestiona que los procesos de enseñanza aprendizaje se ven enriquecidos por las posibilidades que plantean las tecnologías, pero, a la vez, se debe ser consciente de que la educación debe iniciar a las nuevas generaciones a saber vivir e interactuar en un mundo tecnologizado e hiperconectado. Por otra parte, la educación superior atraviesa, pedagógicamente hablando, una etapa orientada a la innovación en la que el influjo tecnológico desempeña un rol indiscutible. Universidades de todas las regiones actualizan y revisan sus metodologías docentes y el papel que juega la innovación y la investigación en sus relaciones con la sociedad. Esto ha supuesto un redescubrimiento de la centralidad de los estudiantes en el proceso educativo, la necesidad de promover aprendizajes más prácticos en las titulaciones, además de desarrollar el compromiso cívico de los estudiantes. De esta forma, muchas universidades han implantado políticas de innovación centradas en el aprendizaje y el desarrollo de competencias. Un ejemplo paradigmático son los programas de aprendizajeservicio que se desarrollan, de forma más o menos institucionalizada, en las instituciones universitarias presenciales y a distancia. Al potencial innovador del aprendizaje-servicio se suma las posibilidades transformadoras de las tecnologías digitales en la educación superior. Nuestro objetivo con este monográfico no es otro que analizar las dinámicas e interacciones 
que se producen entre ambos mundos: entornos virtuales, tecnologías digitales y aprendizajeservicio.

Palabras clave: TIC; educación a distancia; educación virtual; aprendizaje servicio; compromiso ético; educación superior.

\begin{abstract}
Information and communication technologies have transformed our way of seeing and acting in the world, which has led to an unprecedented upheaval in the education sector. Nobody questions that teaching-learning processes are enriched by the possibilities posed by technologies, but, at the same time, it must be aware that education must initiate new generations to know how to live and interact in a technological and hyper-connected world. On the other hand, higher education goes through, pedagogically speaking, a stage oriented to innovation in which the technological influence plays an indisputable role. Universities from all regions update their teaching methodologies and the role of innovation and research in their relations with society. Among other things, this has meant a rediscovery of the centrality of students in the educational process, the need to promote more practical apprenticeships in the qualifications and that, in addition, develop the civic commitment of the students. In this way, many universities have implemented innovation policies focused on learning and the development of competencies where the social responsibility of the University itself with the community plays an important role. A paradigmatic example of all this is the servicelearning programs that are developed, in a more or less institutionalized way, in face to face and distance universities. Our objective with this special issue is none other than to analyze the dynamics and interactions that take place between both worlds: virtual environments, digital technologies and service-learning.
\end{abstract}

Keywords: ICT; distance education; virtual education; service learning; citizenship responsibility; higher education.

Hablar de tecnología es hablar también del ser humano. De ahí que el principal desafío tanto tecnológico como pedagógico sea la humanización de los escenarios digitales de aprendizaje. Desde que existe, ha elaborado e inventado todo tipo de instrumentos, ha desarrollado destrezas, conocimientos que han permitido moldear su entorno para resolver, en primer lugar, sus necesidades y, posteriormente en una segunda fase, atender su curiosidad, ofrecer alternativas a la satisfacción de esas mismas necesidades, considerar nuevos problemas, o abrir nuevas perspectivas. En cada época histórica la tecnología ha estado presente, introduciendo técnicas nuevas e innovadoras para cada momento que, bien han facilitado alguna tarea, han aportado un avance en determinados modos de trabajar, producir o relacionarse; bien han supuesto una transformación radical para la humanidad posibilitando, de este modo, un cambio de era. Así ha sucedido con la utilización de los metales, el fuego, la imprenta, las máquinas de vapor, la electricidad o los superconductores, hasta 
llegar al momento actual con la eclosión del mundo digital. No estamos viviendo algo extraño, ni ajeno, ni novedoso para el ser humano, aunque lo experimentemos de esta forma. Más bien nos encontramos ante una propuesta innovadora que responde al entorno en el que vivimos, a la vez que promueve nuevas situaciones y exigencias.

Ahora bien, debemos resaltar que este momento supone un "algo más" que una innovación, ya que todo lo que han ido generando los avances informáticos, desde sus inicios en la década de los sesenta del pasado siglo, ha favorecido un cambio de paradigma del que aún ahora nos encontramos solo a las puertas. Innovaciones tecnológicas que no se refieren únicamente a artefactos sino a contenidos, aplicaciones, servicios, redes, etc., las cuales realmente están transformando la forma de interaccionar, en cualquier actividad humana, con los otros. Este cambio se produce al facilitar la posibilidad de relacionarse independientemente del tiempo y de dónde se esté localizado, rompiendo las clásicas coordenadas de espacio y tiempo que, hasta ahora, dominaban la comunicación humana y el propio sentido de nuestra existencia. A la vez proporcionan el acceso a un enorme caudal de datos e información, que conlleva el poder decidir y actuar desde unos parámetros radicalmente diferentes.

La realidad es que todo esto está modificando nuestra forma de pensar, de comunicarnos, de convivir, en definitiva, de vivir, al incorporar esta tecnología como parte esencial de interacción del ser humano. El resultado para el ámbito pedagógico es que cada individuo podrá adoptar un rol activo de usuario y, paralelamente, productor, lo que se denomina prosumidor, que, en el contexto educativo, va más allá del dominio de lo informático en su concepto más instrumental, algo que está generando un cambio radical en el modo de entender la educación y su diseño (PérezMateo, Romero y Romeu, 2014).

Estamos ante una tecnología que no se entiende sin la información que proporciona y sin la comunicación que es capaz de facilitar. Por primera vez, la transformación afecta a lo más genuino de todo ser humano: su estructura comunicativa y cognitiva que, por primera vez, se desarrolla a través de un lenguaje multimodal, hipertextual y no lineal (Scolari, 2008; Pérez Escoda, 2015) rompe con el escenario habitual y próximo en el que interacciona. Lo significativo de esta nueva estructura comunicativa es que facilita que todos puedan interconectarse con todos, sin un límite espacial o temporal, con una clara pretensión de inteligencia colectiva apoyada en la participación y colaboración (Cobo y Pardo Kuklinski, 2007; Guitert y Pérez-Mateo, 2013). Ahora bien, estas posibilidades no siempre son positivas o son utilizadas adecuadamente por las personas y las corporaciones, como no deja de aparecer recurrentemente en los medios de comunicación, desde el escándalo de Cambridge Analytica al uso de armas letales autónomas (LAWS), por ejemplo.

En suma, ya no se trata únicamente de transmitir y compartir información, sino de transformarla en conocimiento. $Y$, precisamente, será la tecnología la que facilite los artefactos y los soportes para lograrlo a una velocidad y con un volumen de datos hasta ahora inimaginable. Además, el proceso comunicativo clásico se fracciona al 
desaparecer la clásica interacción en el contexto analógico de emisor y receptor. Esta acción dicotómica se rompe en el entorno digital, ya que todos interaccionamos de forma permanente como emisores y receptores, de acuerdo a las necesidades e intereses de cada momento, cambiamos y/o fundimos los roles (Pérez Escoda, 2015), logrando así una mayor interacción participativa y la elaboración colaborativa del conocimiento. En suma, todos producimos, consumimos, difundimos, etc.; entrecruzándose estas acciones de forma permanente, contribuyendo cada uno con información, con aportaciones que colaboran en el desarrollo del conocimiento en todas sus vertientes (tabla 1 ).

Tabla 1. De la lógica de la edición a la lógica de la relación

\begin{tabular}{|l|l|l|}
\hline \multicolumn{1}{|c|}{$\begin{array}{c}\text { Modelo basado en } \\
\text { contenidos } \\
\text { Lógica de la edición }\end{array}$} & \multicolumn{1}{|c|}{$\begin{array}{c}\text { Modelo basado en la } \\
\text { interacción } \\
\text { (values approach) }\end{array}$} \\
\hline $\begin{array}{l}\text { Orientación del } \\
\text { modelo }\end{array}$ & Web 1.o & Web 2.0 y 3.0 \\
\hline Tipo de protección & Basada en el cuidado & $\begin{array}{l}\text { Basada en la responsabilidad } \\
\text { / seguridad }\end{array}$ \\
\hline $\begin{array}{l}\text { Medidas de } \\
\text { protección }\end{array}$ & $\begin{array}{l}\text { Autorregulación } \\
\text { Control paterno }\end{array}$ & Alfabetización mediática \\
\hline Tipo de narrativa & $\begin{array}{l}\text { Lineal/ } \\
\text { vertical }\end{array}$ & $\begin{array}{l}\text { Hipertextual / } \\
\text { Transmedia }\end{array}$ \\
\hline $\begin{array}{l}\text { Actitudes que } \\
\text { promueven }\end{array}$ & $\begin{array}{l}\text { Lectura, observación, } \\
\text { escucha }\end{array}$ & $\begin{array}{l}\text { Interacción, comunicación, } \\
\text { diálogo }\end{array}$ \\
\hline Imagen de la infancia & $\begin{array}{l}\text { Espectador/ } \\
\text { consumidor }\end{array}$ & $\begin{array}{l}\text { Usuario/ } \\
\text { creador online (prosumidor) }\end{array}$ \\
\hline
\end{tabular}

Fuente: García-Gutiérrez, 2015.

Si valoramos esta situación en el contexto educativo, hasta ahora era el profesor (la autoridad) el emisor del conocimiento, quien transmitía los contenidos que los receptores (alumnos) debían asimilar, el que decidía la información a transmitir, por lo que mantenía el control del proceso educativo. Sin embargo, aunque la situación anterior se mantiene formalmente, en el contexto digital el control se desliza también hacia el aprendiz, ya que este es el que accede a la información, selecciona y solicita aquella que más le interesa (y, es también sobre este punto sobre el que incidir pedagógicamente). A la vez, también se puede, junto con otros, colaborar en la construcción de conocimiento nuevo. Así, esta denominada sociedad del conocimiento se erige desde una teoría de la comunicación digital interactiva, cuyo énfasis ya no se pone en la comprensión de los mensajes, los receptores o los 
emisores, sino en "la comprensión de las hipermediaciones generadas en los espacios virtuales" (Pérez Escoda, 2015, p. 60).

En definitiva, estamos ante la convergencia digital de los servicios, redes y dispositivos de la sociedad de la información y de los medios de comunicación, lo que ocasiona la conocida sociedad red, espacio en el que inevitablemente estamos inmersos. Red que no es simplemente tecnología, sino el medio de interacción, de comunicación que configura la forma organizativa de nuestras sociedades (Castells, 2011). De ahí que no se deba perder de vista que lo importante no es la tecnología, ni los avances con los que sorprende día a día, sino el entorno que se está y estamos construyendo. Espacio digital que no puede perder, como entorno en el que se vive, su dimensión humanizadora, ya que "todo lo que es inherente a lo humano es lo más difícil de automatizar: la creatividad, el pensamiento crítico, la inteligencia emocional, la capacidad de inspirar y de trabajar colaborativamente y otras habilidades humanas, (...)" (Escamilla, 2018, pp. 13-14). Competencias que son, precisamente, las que deben ser priorizadas en la formación de toda persona. Capacidades que distingue al ser humano de todo lo meramente tecnológico, por lo que deberán formar parte del "núcleo duro" del aprendizaje en un permanente proceso de aprender a aprender.

Se construye un escenario que, a su vez, está planteando nuevas exigencias a la educación, ya que no se trata de acordar qué nuevas asignaturas y/o contenidos deben ser enseñados en las escuelas, en las universidades o en otros lugares de formación, sino de plantear una nueva forma de lograrla, al afrontar tanto necesidades como procesos de aprendizaje radicalmente diferentes. Además, se trata de aprender en los lenguajes que exige este aprendizaje multimodal e hipertextual en diferentes soportes y en el que debemos ser capaces tanto de leer en diferentes pantallas, en distintos formatos (alfabético, alfanumérico, digital, icónico, visual, plástico, etc.), como de comunicarnos a través de ellos. Dominar las competencias comunicativas, mediáticas y digitales serán claves para poder participar en esta sociedad. Sin duda, las competencias informacionales, cognitivas y éticas facilitarán el poder afrontar el alto volumen de información y de conocimiento disponible necesario para afrontar y resolver, cada día, las situaciones personales, profesionales, comunitarias, etc. y, también, saber dilucidar y hacer frente a los desafíos éticos que las tecnologías digitales nos deparan.

\section{SIN PERDER DE VISTA LA PLENITUD HUMANA: EL (RE)DISEÑO DE LOS NUEVOS ESPACIOS Y PROCESOS DE APRENDIZAJE}

Como decíamos al inicio de la presentación, "hablar de tecnología es hablar también del ser humano", de ahí que no podamos perder de vista el desarrollo de la plenitud humana cuando hablamos de la progresiva aplicación de las tecnologías digitales a los procesos educativos. Uno de los escenarios que más se ha beneficiado de estas propuestas es la educación a distancia y/o virtual, que vive en estas décadas 
una expansión sin precedentes (García Aretio y Ruiz-Corbella, 2015). Sería simplificar mucho el afirmar que esta expansión se debe a la evolución tecnológica, ahora sin ella no habría sido posible. Sin duda, los avances en las ciencias computacionales, tecnológicas, físicas, matemáticas, psicológicas o pedagógicas, junto a factores políticos, económicos y sociales, han favorecido esta revolución en el modo de afrontar el aprendizaje y, en consecuencia, en el modo de enseñar. El gran paso, que se ha producido, es que se ha superado

(...) el sentido artefactual y simplificado de la tecnología educativa, referido solo a las herramientas que sirven para enseñar y aprender, y se construye una versión más compleja que incluye a todos los elementos humanos y no humanos en procesos dinámicos (DeLanda, 2006; Usher y Edwards, 2005). Lo anterior obliga a construir un nuevo relato epistemológico para el estudio de los escenarios educativos tecnologizados, que se caracterizan por estar pletóricos de elementos heterogéneos que mantienen distintas conexiones entre sí, como el software, las redes sociales, el hardware, la señal de internet, la inteligencia artificial, la inteligencia aumentada, el internet de las cosas, las plataformas de gestión del aprendizaje y, por supuesto, también los humanos (Escudero Nahón, 2018, p. 154).

No hay duda de que estamos ante lo que se está denominando "cuarta revolución industrial”, que se ha generado a partir de ecosistemas digitales capaces de interconectar consumidores, máquinas, productos y servicios. Se ha producido, de nuevo, un proceso disruptivo con todo lo que conocemos hasta ahora. Ejemplos de este nuevo escenario son Amazon como modelo de negocio, Google de información o Netflix de entretenimiento. Todos ellos facilitan una forma de localizar, recuperar, seleccionar, utilizar, transformar, aplicar datos de forma personalizada, algo que exige de competencias personales, profesionales y ciudadanas diferentes $\mathrm{o}$, al menos, "evolucionadas" en relación a las atendidas hasta ahora por toda institución educativa.

Ahora, a pesar de que lo tecnológico es la clave en esta cuarta revolución, se debe tener claro que no toda propuesta tecnológica logra un resultado innovador por sí sola, ni el diseño de varias acciones online conduce a una transformación del aprendizaje. Es decir, incluir un ipad o una pizarra digital no constituye por sí mismo una innovación pedagógica. Este maremágnum de propuestas tecnológicas, de innovaciones o de nuevas formas de enseñar nos lleva a plantear una clasificación que ayude a diferenciar entre aquello que proyecta una evolución, una innovación o supone una auténtica revolución educativa. Por ello, es importante analizarlas, clasificarlas, distinguir las soluciones e identificar sus impactos (tabla 2) para poder valorar la influencia real de cada una de estas y su capacidad de transformar la realidad. 
Tabla 2. Niveles e incidencia pedagógica del impacto de la innovación educativa

\begin{tabular}{|l|l|l|l|}
\hline \multicolumn{1}{|c|}{ Proceso } & \multicolumn{1}{|c|}{$\begin{array}{c}\text { Aplicación } \\
\text { metodológica }\end{array}$} & \multicolumn{1}{c|}{ Impacto } & \multicolumn{1}{c|}{$\begin{array}{c}\text { Uso de la } \\
\text { tecnología }\end{array}$} \\
\hline \multirow{2}{*}{ Evolución } & Sustitución & \multirow{2}{*}{ Mejora } & Integración básica \\
\cline { 2 - 4 } & Aumento & & Inclusión intencional \\
\hline Innovación & Modificación & Transformación & $\begin{array}{l}\text { Inmersión } \\
\text { tecnológica }\end{array}$ \\
\hline Revolución & Redefinición & &
\end{tabular}

Fuente: adap. de Escudero Nahón, 2018; Ostrowicz, 2018

En la mayoría de las propuestas educativas, los cambios que se introducen son por evolución. Se incorporan nuevos recursos, sustituyendo otros que aportan elementos originales, lo que contribuye con un modo de trabajar que atrae por la novedad, pero que no llega a impactar. Al tiempo de su utilización se evidencia que no originan mejoras en el aprendizaje, ya que no logra una transformación de las prácticas habituales de trabajo. Un ejemplo de lo anterior es la utilización de Wikipedia, la inclusión de documentación en línea o grabaciones de Youtube, como recursos de aula. En todos ellos se da una integración básica. Se han sustituido los recursos habituales por recursos en línea, pero el diseño sigue siendo el mismo y la interacción profesor-estudiantes, o estudiantes con sus pares, continúa de la misma forma. Sin duda, es factible la mejora en rendimiento, en resultados o en la eficacia de determinados aprendizajes, pero no existe transformación en el diseño pedagógico. Otro ejemplo de esta evolución es la utilización de Facebook por parte de los estudiantes universitarios. Se ofrece en estas páginas una participación y una interacción significativa entre ellos, pero no elaboran conocimiento, ni desarrollan un proyecto común, ya que se utiliza como espacio de intercambio de trabajos, resúmenes, exámenes, o punto de reunión para organizar reclamaciones. Se utiliza una tecnología muy potente con el mismo esquema de interacción que, hasta ahora, se desarrollaba en pasillos o en la cafetería de cada universidad. Únicamente se ha sustituido un espacio por otro.

En cambio, en los procesos innovadores lo que se pretende es una modificación sustancial de lo que se ha venido haciendo, al introducir los elementos tecnológicos para un mejor diseño instruccional, para lograr mejores resultados formativos, para afianzar el camino hacia una auténtica transformación. Necesitamos propuestas innovadoras para romper con dinámicas y modos de hacer, para experimentar nuevos modos de desarrollar procesos y proyectos educativos. El paso de la educación a distancia a la educación virtual, con todos los recursos tecnológicos que se han utilizado, fue un proceso innovador. Las grandes diferencias entre unas propuestas formativas y otras estriban en si se ha limitado a una sustitución de recursos o a un aumento que incide, de una u otra forma, en el diseño instruccional, o si realmente se ha originado una modificación en ese proceso. Como ejemplo, un MOOC (Massive 
open online course) puede resultar una propuesta innovadora al modificar el diseño instruccional en la que se facilita el trabajo colaborativo, la participación en la elaboración del conocimiento, el trabajo apoyado en experiencias reales, la interacción entre todos los participantes, etc. Se da una inclusión intencional de tecnologías que facilitan y logran modificar formas de aprendizaje. En esta línea nos encontramos con muchos proyectos innovadores de gran interés, en el que exponen la inclusión de la gamificación en el aula, de la geolocalización, de los storytelling, de la realidad virtual, de nuevas formas de evaluación, etc. Son experiencias puntuales que, bien se han llevado a cabo en un tiempo determinado, no teniendo continuidad; bien se desarrollan en una asignatura determinada sin llegar a influir y transformar el proyecto formativo de la titulación o del curso en la que se imparte.

Por último, estaría el proceso revolucionario que está generando propuestas de aprendizaje virtual (e-learning), de entorno personal de aprendizaje, entorno virtual de enseñanza-aprendizaje, educación mediada por tecnología, aprendizaje en red, etc., apoyadas en avances tecnológicos como la inteligencia artificial, el big data o el cloud computing, que todas ellas exigen una transformación radical del modo de pensar y diseñar cada proceso formativo. No se trata de incluir estos recursos, sino de diseñar los procesos de aprendizaje inmersos en estos escenarios digitales, de pensar la formación de acuerdo a esa nueva realidad en la que se interacciona en todas las actividades humanas. En este proceso la formación ya no está limitada a un escenario específico, como puede ser el aula de una universidad, sino que todo contexto debe ser contemplado como un recurso y un espacio para la formación. En este escenario es necesario formar en competencias, especialmente las genéricas (soft skills), las cuales facilitan la capacidad de localizar, recuperar y utilizar la información, los datos que necesitamos en cada caso; a ser capaces de analizar, de elaborar conocimiento, de participar en esa construcción con otros iguales. Y no solo se está hablando de la utilización de determinadas tecnologías, más o menos sofisticadas, sino que estas se entrelazan con los avances de otras ciencias -la psicología cognitiva, la pedagogía, la lingüística, etc.- logrando una auténtica transformación del proceso formativo. Un primer paso de esta transformación, que para muchos pasa inadvertida, es el cambio de lenguaje: ya no hablamos del proceso de enseñanza, ni de enseñanza-aprendizaje, sino del proceso de aprendizaje.

En este contexto y con el objetivo de abordar esta temática, este monográfico, (...) Aprendizaje-servicio y tecnologías digitales: un desafio para los espacios virtuales de aprendizaje, reúne siete artículos que abordan el aprendizaje-servicio (ApS) como metodología innovadora para la educación superior, con la particularidad de afrontarla desde la perspectiva de su inclusión y desarrollo en escenarios virtuales de aprendizaje. Como punto de partida, todos los autores y autoras, procedentes de cuatro países con tradiciones pedagógicas muy diferenciadas, como son Argentina, Estados Unidos, Venezuela y España, confirman el nuevo escenario educativo que, poco a poco, se está imponiendo apoyado en las innumerables opciones que facilita la tecnología, además del afianzamiento del paradigma en el que el aprendizaje es 
el eje de todo diseño de formación. Justamente, un acierto del monográfico ha sido la diversidad de trabajos que lo compone, ya que refleja no solo el interés del tema a nivel internacional sino la particular aportación de cada trabajo según la región del globo donde se sitúan los autores.

Con estas coordenadas, tecnología y aprendizajes, se plantean diversas experiencias desde diferentes enfoques, con la propuesta de la metodología del ApS en los escenarios digitales de educación superior.

Sobre ApS y su aplicación en las instituciones de educación superior se ha escrito mucho, en cambio resulta novedoso e innovador, especialmente, en la región iberoamericana, desarrollar esta particularidad metodología en entornos digitales de aprendizaje. Favorecer proyectos de innovación docente sobre aprendizajeservicio en escenarios virtuales aporta experiencias y conocimientos necesarios para desarrollar metodologías innovadoras en las que se une de forma real los aprendizajes teóricos con la aplicación práctica en entornos auténticos apoyados en las posibilidades que facilitan las tecnologías de la información y la comunicación.

Con este objetivo este monográfico comienza con el artículo elaborado por Richard Bringle y Patti Clayton, del Center for Service and Learning de Indiana University and Purdue University (USA), en el que presentan, a modo introductorio, un marco amplio en el que reflexionan sobre las temáticas objeto de estudio en el monográfico. Es importante, cómo hacen notar estos autores, cobrar conciencia del desarrollo global que actualmente tiene el ApS, y cómo este desarrollo global se va concretando en diversas regiones geográficas, lo que diversifica las denominaciones y las características de los proyectos, de acuerdo a las particularidades culturales y geográficas de cada región. Además, este proceso se enriquece con la integración de las tecnologías digitales en los propios proyectos de ApS.

Por su parte, Miguel Ángel Santos Rego, Igor Mella Núñez y Alexandre Sotelino, de la Universidad de Santiago de Compostela (España), valoran la presencia de la internacionalización en las nuevas metodologías que se están introduciendo en las universidades. Para ello llevan a cabo una revisión bibliográfica a partir de los descriptores 'aprendizaje-servicio internacional' y 'aprendizaje-servicio electrónico', en el que comprueban que esta metodología está dando respuesta a las tendencias pedagógicas que mayor impacto están teniendo en la educación superior.

Ángeles Manjarrés, Simon James Pckin, Héctor Alonso Meana y Natalia Rodríguez-Fernández, de la Universidad Nacional de Educación a Distancia y de la Universidad Complutense (España), presentan el desarrollo (aún en fase experimental) de una aplicación web, Virtu@al-ApS, elaborada de forma conjunta por estudiantes del Grado en Educación Social y de Ingeniería Informática dirigida a dar apoyo a estos proyectos innovadores.

Por su parte, María Rosa Tapia, del Centro Latinoamericano de Aprendizaje Servicio - CLAYSS (Argentina), presenta una investigación sobre la inclusión de las tecnologías digitales en los proyectos de aprendizaje y servicio solidario a partir del análisis de las tecnologías de la información y de la comunicación (TIC) en el 
desarrollo de proyectos de ApS en escuelas secundarias públicas de Argentina. En la investigación se analizan una serie de proyectos en los que las TIC se integraron en las diferentes disciplinas para "ampliar" la experiencia de los estudiantes y ayudarlos a conectar el conocimiento "distribuido" a la resolución de problemas concretos de su comunidad.

Continuando en esta región, Beatriz Elena Sandia y Jonás Arturo Montilba C., de la Universidad de Los Andes (Venezuela), exponen los resultados de un análisis documental de las competencias y valores que caracterizan a la ciudadanía del nuevo milenio y cómo el aprendizaje-servicio juega un papel clave en la formación ciudadana, lo que va a servir para elaborar un modelo para la comprensión de los espacios virtuales para el aprendizaje-servicio.

Juan Luis Fuentes, Prado Martín-Ondarza y Paloma Redondo Corcobado, de la Universidad Complutense (España), apuntan como el espacio físico y el virtual desempeñan un papel central e interrelacionado en proyectos de ApS, en el que la comprensión del papel de las tecnologías en el ejercicio de la ciudadanía y en el desarrollo de proyectos pedagógicos innovadoras resulta crucial.

Anna Escofet, de la Universitat de Barcelona (España), analiza en su trabajo la relación entre tecnologías digitales y ApS. En efecto, el uso generalizado de las tecnologías digitales y la extensión de las redes sociales genera espacios de comunicación y encuentro en los que las personas y actores sociales y políticos entran en interconexión. Estas conexiones abren espacios y posibilidades diversas de participación digital, de manera más accesible, instantánea, continuada, igualitaria y personalizada. De esta forma, unir las tecnologías digitales con proyectos de ApS facilita que jóvenes y niños experimenten de forma real aquello que es la solidaridad, la responsabilidad y el servicio a los otros, así como la satisfacción por los objetivos conseguidos. En definitiva, facilita conseguir el desarrollo de una ciudadanía responsable y consciente.

Por último, Marta Ruiz-Corbella y Juan García-Gutiérrez de la Universidad Nacional de Educación a Distancia - UNED (España) muestran una experiencia de ApS virtual que integra las tecnologías en su diseño y desarrollo generando una modalidad de ApS inmersiva y real en el ciberespacio. Con esta experiencia evidencian que no solo es posible tratar estas metodologías en estos escenarios virtuales, sino que estas multiplican sus posibilidades y efectos pedagógicos.

Distintas formas de ver y aplicar una misma realidad pedagógica, diferentes modos de afrontar un contexto emergente. Tecnología y educación están llamadas a encontrarse en fórmulas innovadoras que abran perspectivas formativas necesarias en este nuestro siglo XXI. Y, sin duda, una de estas fórmulas es el ApS virtual. 


\section{REFERENCIAS}

Castells, M. (2011). Democracy in the age of the Internet. Transfer: Journal of contemporary culture, 6, 96-103. Recuperado de https://bit.ly/2PqzH3D

Cobo, C., y Pardo Kuklinski, H. (2007). Planeta Web 2.o. Inteligencia colectiva o medios fast food. Grup de Recerca d'Interaccions Digitals. Barcelona / México DF.: Universitat de Vic, Flacso.

Escamilla, J. (2018). El valor de enseñar para el mañana. Telos, 11o, 10-15. Recuperado de https://bit.ly/2GtC5pb

Escudero Nahón, A. (2018). Redefinición del "aprendizaje en red" en la cuarta revolución industrial. Apertura, 10(1), 149-163. doi: http://dx.doi.org/10.18381/ Ap.v10n1.1140

García Aretio, L., y Ruiz-Corbella, M. (2015). Educación a distancia, la perspectiva de la universidad en una sociedad en red: Una perspectiva desde las regiones de América Latina, El Caribe y América del Norte. Revista Española de Educación Comparada, 26, 11-14. doi: 10.5944/ reec.26.2015. 15819.

García-Gutiérrez, J. (2015). El modelo europeo de protección a la infancia en internet. Del cuidado a la responsabilidad. Teoría de la Educación. Revista Interuniversitaria, 27(1), 119-136. doi 10.14201/teoredu2015271119136.
Guitert, M. y Pérez-Mateo, M. (2013). La colaboración en la red: hacia una definición de aprendizaje colaborativo en entornos virtuales. Teoría de la Educación. Educación y Cultura en la Sociedad de la Información, 14(1), 10-31. Recuperado de https://bit.ly/2kowhev

Ostrowicz, I. (2018). Lo que se esconde detrás de la EdTech y la LearnTech. Telos, 110, 30-35. Recuperado de https://bit. ly/2JRRO39

Pérez Escoda, A. (2015). Alfabetización digital y competencias digitales en el marco dela evaluación educativa:Estudio en Docentes y Alumnos de Educación Primaria en Castilla y León. [Tesis Doctoral]. Salamanca: Universidad de Salamanca. Recuperado de https://bit. ly/2lwZKgY

Pérez-Mateo, M., Romero, M., y Romeu, T. (2014). La construcción colaborativa de proyectos como metodología para adquirir competencias digitales. Comunicar, 42, 15-24. doi: https://doi.org/10.3916/C422014-01

Scolari, C. (2008). Hipermediaciones: elementos de una teoría de la comunicación digital interactiva. Barcelona: Gedisa.

\section{PERFIL ACADÉMICO Y PROFESIONAL DE LOS AUTORES}

Juan García-Gutiérrez. Profesor Contratado. Doctor de Filosofía de la Educación de la Universidad Nacional de Educación a Distancia (España). Coordinador del Grupo de Innovación Docente para el Desarrollo de la Competencia Ética y Cívica en la Educación Superior. Delegado del Rector de la Oficina de Aprendizaje-Servicio de la UNED. Entre sus temas de interés destacan la filosofía de la educación, educación moral, tecnologías y educación ética.

E-mail: juangarcia@edu.uned.es 
Marta Ruiz-Corbella. Profesora Titular de la Universidad Nacional de Educación a Distancia (España). Miembro del Grupo de Investigación Educación Superior Presencial y a Distancia (ESPYD) y del Grupo de Innovación Docente para el Desarrollo de la Competencia Ética y Cívica en la Educación Superior. Entre sus temas de interés destacan la educación moral y cívica, la educación superior, la formación del profesorado y la educación a distancia. ID: https://orcid.org/oooo0001-5498-4920

E-mail: mruiz@edu.uned.es

Dirección:

Dpto Teoría de la Educación y Pedagogía Social

Facultad de Educación - UNED

c/ Juan del Rosal, 14

28040 Madrid (España)

Fecha de recepción del artículo: 22/08/2019

Fecha de aceptación del artículo: 22/08/2019

Fecha de aprobación para maquetación: 13/10/2019 